\title{
A null mutation in ANGPTL8 does not associate with either plasma glucose or type 2 diabetes in humans
}

\author{
Katharine R. Clapham ${ }^{1}$, Audrey Y. Chu, ${ }^{2,3}$ Jennifer Wessel ${ }^{4,5}$, Pradeep Natarajan 1,6,7,8, Jason Flannick ${ }^{8,9}$, \\ Manuel A. Rivas ${ }^{1,8}$, Samantha Sartori ${ }^{10}$, Roxana Mehran ${ }^{10}$, Usman Baber ${ }^{10}$, Valentin Fuster ${ }^{10}$, Robert A. Scott ${ }^{11}$, \\ Daniel J. Rader ${ }^{12}$, Michael Boehnke ${ }^{13}$, Mark I. McCarthy ${ }^{14,15,16}$, David M. Altshuler ${ }^{8,9,17}$, Sekar Kathiresan 1,6,7,8 \\ and Gina M. Peloso $1,6,7,8,18^{*}$
}

\begin{abstract}
Background: Experiments in mice initially suggested a role for the protein angiopoietin-like 8 (ANGPTL8) in glucose homeostasis. However, subsequent experiments in model systems have challenged this proposed role. We sought to better understand the importance of ANGPTL8 in human glucose homeostasis by examining the association of a null mutation in ANGPTL8 with fasting glucose levels and risk for type 2 diabetes.

Methods: A naturally-occurring null mutation in human ANGPTL8 (rs145464906; c.361C > T; p.Q121X) is carried by 1 in 1000 individuals of European ancestry and is associated with higher levels of plasma high-density lipoprotein cholesterol, suggesting that this mutation has functional significance. We examined the association of p.Q121X with fasting glucose levels and risk for type 2 diabetes in up to 95,558 individuals (14,824 type 2 diabetics and 80,734 controls).

Results: We found no significant association of p.Q121X with either fasting glucose or type 2 diabetes ( $p$-value $=0.90$ and 0.65 , respectively). Given our sample sizes, we had $>98 \%$ power to detect at least a $0.23 \mathrm{mmol} / \mathrm{L}$ effect on plasma glucose and $>95 \%$ power to detect a $70 \%$ increase in risk for type 2 diabetes.

Conclusion: Disruption of ANGPTL8 function in humans does not seem to have a large effect on measures of glucose tolerance.
\end{abstract}

Keywords: Betatrophin, Angiopoietin-like 8, rs145464906

\section{Background}

In type 1 and to a lesser extent in type 2 diabetes, the insulin-producing beta cells of the pancreas undergo destruction and exhibit decreased function. Recovered insulin production by abrogating this process has important therapeutic implications for individuals suffering from diabetes mellitus. A study in mice demonstrated that pancreatic beta cell proliferation is stimulated by a 198-amino acid protein named betatrophin (also known as Lipasin

\footnotetext{
* Correspondence: gpeloso@bu.edu

'Department of Medicine, Massachusetts General Hospital, Harvard Medical School, Boston, MA 02114, USA

${ }^{6}$ Center for Human Genetic Research, Massachusetts General Hospital,

Boston, MA 02114, USA

Full list of author information is available at the end of the article
}

and ANGPTL8), leading to improved glucose tolerance [1]. Injection of betatrophin-expressing plasmids into mice resulted in increased beta cell replication and ultimately increased beta cell number and pancreatic beta cell mass. However, subsequent murine experiments of betatrophin have largely been neutral with respect to glucose alterations and overexpression of ANGPTL8 showed no abnormalities in glucose homeostasis [2, 3]. In addition, Angptl8 $8^{-/}$mice did not demonstrate impaired glucose tolerance [2, 4]. Although its role in glucose homeostasis is still being elucidated, ANGPTL8 is known to have a role in the regulation of lipid metabolism, possibly through activation of a related protein, angiopoietin-like 3 [5]. Several experiments have shown that Angptl8 null mice have lower triglyceride levels and conversely, that 
overexpression of ANGPTL8 increases triglyceride levels $[2,4,5]$. Taken together, findings in model systems suggest that whereas a role for ANGPTL8 in beta cell proliferation is uncertain, ANGPTL8 is consistently linked to triglyceride concentrations.

Null mutations can be used to inform on the effect of a gene in humans. We have previously reported a naturally-occurring null mutation in human ANGPTL8 (rs145464906; c.361C > T; p.Q121X). ANGPTL8 p.Q121X is estimated to be carried by 1 in 1000 individuals of European ancestry and 1 in 10,000 African-Americans [6]. Plasma high-density lipoprotein cholesterol is $10 \mathrm{mg} / \mathrm{dl}$ higher $\left(p\right.$-value $\left.=5 \times 10^{-11}\right)$ and triglyceride concentration is $15 \%$ lower $(p$-value $=0.003)$ in carriers of ANGPTL8 p.Q121X [6].

The lipid phenotype demonstrated by carriers of ANGPTL8 p.Q121X suggests that this naturally occurring mutation has functional significance and allows us to test the hypothesis that carrying ANGPTL8 p.Q121X also perturbs glucose homeostasis in humans. Previously, we have shown that ANGPTL8 p.Q121X was not associated with fasting glucose in two studies $(\mathrm{n}<15,000)$ [6]. Here, we aimed to determine the association of ANGPTL8 p.Q121X with two measures of glucose homeostasis fasting glucose level and type 2 diabetes status in up to 95,558 individuals (14,824 type 2 diabetics and 80,734 controls).

\section{Methods}

\section{In silico analysis}

In silico analysis was performed to predict the effect of ANGPTL8 p.Q121X (rs145464906) mutation on transcription. We predicted whether the ANGPTL8 p.Q121X mutation resulted in nonsense mediated decay using RNA sequencing transcript isoform data publically available from the Genotype-Tissue Expression (GTEx) project (http://www.gtexportal.org/) and a predictive model implemented in MAMBA (http://www.well.ox.ac.uk/ rivas/ mamba) $[7,8]$.

\section{Study participants}

Analysis was limited to subjects of self-reported European ancestry. Studies contributing summary results included the BioImage Study [9], the Cohorts for Heart and Aging Research in Genomic Epidemiology (CHARGE) Consortium Diabetes Working Group [10], and studies from the T2D-GENES consortium with $>5$ minor alleles for ANGPTL8 p.Q121X (KORA and UK) [11]. The BioImage Study and the KORA and UK studies from the T2DGENES consortium have been approved by the MIT IRB (\#1010004095, \#0912003615 \#1107004579, respectively). The CHARGE Diabetes Working Group consists of 27 European Ancestry studies and all participating studies were approved by local institutional review committees. All subjects have provided written informed consent.

\section{Genotyping and quality control}

All study participants were genotyped on the HumanExome BeadChip v.1.0 or v1.1 (Illumina) for ANGPTL8 p.Q121X and called using joint calling [12], GenomeStudio or zCall [13]. Quality control involved checking concordance to GWAS data and excluding those individuals missing $>5 \%$ genotypes, population clustering outliers, individuals with high inbreeding coefficients or heterozygote rates, individuals with gender mismatches, one individual from duplicate pairs, and individuals with an unexpectedly high proportion of identity-by-descent sharing, with consideration for family studies, based on high-quality variants. All contributing studies used an additive coding of variants to the minor allele.

\section{Phenotypes}

Association analyses were performed for fasting glucose and type 2 diabetes individually by cohort and summary statistics were shared. In the Biolmage Study, type 2 diabetes was defined as individuals taking a medication for diabetes, having a fasting glucose $>126 \mathrm{mg} / \mathrm{dl}$ or having been told that he/she had diabetes. Type 2 diabetes was defined in the CHARGE Consortium Diabetes Working Group according to Wessel et al [10], and in the T2DGENES Consortium according to Voight et al [14]. Individuals with a diagnosis of diabetes were excluded from the fasting glucose and insulin analyses to avoid the variable effects of diabetes medications on the quantitative traits.

\section{Statistical analyses}

Two primary analyses were performed separately by study in available participants: (1). linear regression of fasting glucose levels with an additive coding of ANGPTL8 p.Q121X adjusting for age, sex, and principal components of ancestry; and (2). logistic regression of type 2 diabetes with an additive coding of ANGPTL8 p.Q121X adjusting for age, sex, and principal components of ancestry. Counts of the number of ANGPTL8 p.Q121X mutation carriers were obtained in type 2 diabetes cases and controls. Additionally, in 47,388 we analyzed the association of ANGPTL8 p.Q121X with fasting insulin levels adjusted for BMI, age, sex, and principal components of ancestry.

For the outcomes of fasting glucose and fasting insulin levels, statistical evidence from each study was combined using fixed-effects inverse variance meta-analysis. Heterogeneity of effects between studies was ruled out $(\mathrm{p}>0.05)$.

For the outcome of type 2 diabetes, we performed meta-analyses by using Cochran-Mantel-Haenszel statistics for stratified 2x2 tables. The Cochran-Mantel-Hanszel method combines score statistics rather than Wald 
statistics. As an alternate approach, we performed a fixedeffects inverse variance meta-analysis on the betas and standard errors from the individual study results. Using a fixed-effects inverse variance meta-analysis did not change the results.

All analyses were performed using the software program $\mathrm{R}$ version 2.15. We estimated statistical power to detect association of ANGPTL8 p.Q121X with fasting glucose and type 2 diabetes using the Genetic Power Calculator (http://pngu.mgh.harvard.edu/ purcell/gpc/) [15].

\section{Results}

The lipid phenotype previously demonstrated by carriers of ANGPTL8 p.Q121X suggests that this naturally occurring mutation has functional significance. In addition, using in silico predictions, we found that ANGPTL8 p.Q121X results in nonsense mediated decay of the RNA transcript and partial knockdown of the resulting protein (Additional file 1: Figure S1).

All participants were of self-reported European ancestry. 69,854 individuals without type 2 diabetes were available for the analysis of fasting glucose levels. In analysis for type 2 diabetes status, 14,824 individuals with a diagnosis of type 2 diabetes were compared to 80,734 controls (Additional file 1: Table S1). 24 (0.16\%) of the type 2 diabetes cases were heterozygous carriers of the minor allele for ANGPTL8 p.Q121X whereas $142(0.18 \%)$ of the controls were heterozygous carriers.

We combined evidence from all available studies and performed a fixed-effects meta-analysis for the association of p.Q121X with fasting glucose levels. We found no evidence for association of p.Q121X with fasting glucose levels (beta $=0.002 \mathrm{mmol} / \mathrm{L} ; 95 \%$ conference interval $[\mathrm{CI}]=(-0.025,0.029) ; p$-value $=0.90) \quad($ Table 1$)$. For the outcome of type 2 diabetes, we used CochranMantel-Haenszel meta-analysis to combine the counts across the studies given the low number of type 2 diabetes cases with the p.Q121X mutation. We found no evidence for association of p.Q121X with type 2 diabetes (Fig. $1, \mathrm{OR}=0.88,95 \% \mathrm{CI}=0.57-1.36, p$-value $=0.65$ ).

Additionally, we found no evidence of association for fasting insulin levels adjusted for BMI with ANGPTL8 p.Q121X in 47,388 individuals with fasting insulin available within CHARGE (3 \% change; $p=0.43$ ).

We estimated statistical power to detect each of these associations given our analyzed sample sizes. Power

Table 1 Association of ANGPTL8 p.Q121X with plasma glucose

\begin{tabular}{llccl}
\hline Study & $\mathrm{N}$ & Effect size & Standard error & $P$ \\
\hline Biolmage & 4,072 & 0.080 & 0.19 & 0.70 \\
CHARGE & 49,838 & -0.005 & 0.05 & 0.92 \\
T2D-GENES & 15,944 & 0.002 & 0.01 & 0.89 \\
Meta-analysis & 69,854 & 0.002 & 0.01 & 0.90 \\
\hline
\end{tabular}

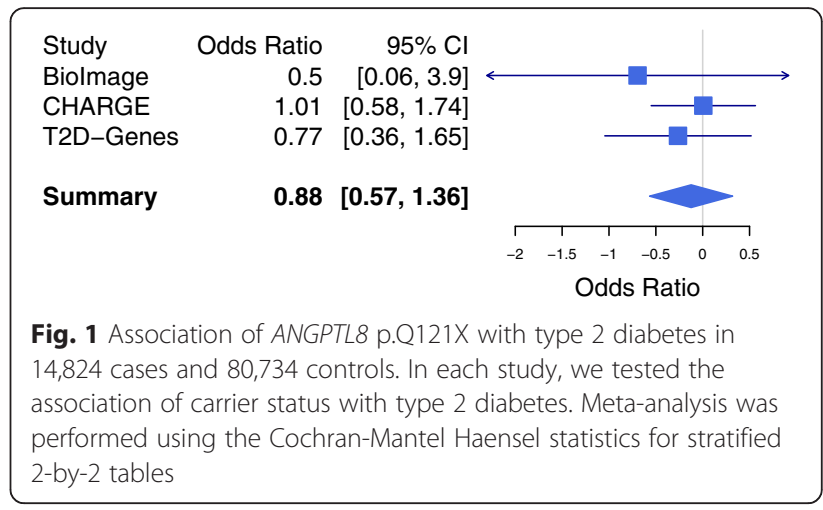

calculations indicate that with a sample size of 69,854 we had $>98 \%$ power to detect a $0.23 \mathrm{mmol} / \mathrm{L}$ (1/2-standard deviation; $4.2 \mathrm{mg} / \mathrm{dL}$ ) effect of p.Q121X on fasting glucose at an alpha level of 0.05 . Given 14,824 type 2 diabetes cases and 80,734 controls, we have $>95 \%$ power to detect a genotype relative risk for carriers of $>1.7$ and $>$ $85 \%$ power to detect a genotypic relative risk for carriers of $>1.6$ at an alpha level of 0.05 .

\section{Discussion}

We find that carrying a null mutation in human ANGPTL8 is neither associated with fasting glucose nor risk for type 2 diabetes in analyses involving up to 95,558 individuals. These results are consistent with findings from a preliminary analysis of individuals carrying ANGPTL8 p.Q121X with fasting glucose in Peloso, et al [6], in which no significant association between ANGPTL8 p.Q121X and fasting glucose levels was identified in $<15,000$ individuals, which only had $45 \%$ power to detect a $0.23 \mathrm{mmol} / \mathrm{L}$ (1/2-standard deviation; $4.2 \mathrm{mg} /$ dL) effect of p.Q121X on fasting glucose at an alpha level of 0.05 . However, the larger sample size utilized in our study allowed for $98 \%$ power and a better estimation of the impact of ANGPTL8 p.Q121X on fasting glucose levels. This study also expands upon the phenotyping of the carriers of this allele in that we were able to examine association of ANGPTL8 p.Q121X with a diagnosis of type 2 diabetes and with fasting insulin levels, and to ask a targeted hypothesis that may not reach an exome chip-wide significance level $\left(p<3 \times 10^{-7}\right.$ for fasting glucose and $p<4.5 \times 10^{-7}$ for type 2 diabetes) as was explored in Wessel, et al [10].

While our results do not support the role of ANGPTL8 inhibitors for treatment of type 2 diabetes, there remains evidence that ANGPTL8 inhibition remains a viable anti-triglyceride target $[2,6]$. The human genetic analysis presented here provides evidence that an ANGPTL8 inhibitor aimed at lowering plasma triglyceride levels will not have a major effect on glucose tolerance in humans. 
Some limitations deserve mention. The initial therapeutic hypothesis from model systems related to gain of gene function; loss of gene function as assessed by p.Q121X may not necessarily lead to phenotypic effects in a direction opposite to gain of gene function. In addition, due to the scarcity of the allele, we were able to assess only heterozygous carriers of the allele. It is possible that there is a recessive effect on fasting glucose levels or type 2 diabetes status that would be unmasked only in individuals homozygous for the ANGPTL8 mutation. The scarcity of the null mutation also limits our statistical power, and we are unable to rule out modest effects on glucose and type 2 diabetes.

\section{Conclusion}

While ANGPTL8 has been shown to robustly associate with plasma triglyceride levels, our results do not support a role for ANGPTL8 in glucose homeostasis in humans.

\section{Additional file}

\section{Additional file 1: Supplemental Information. Figure S1. In-silico}

predictions of the impact of premature termination codon p.Q121X suggests that the variant would trigger nonsensemediated decay. Table S1. ANGPTL8 p.Q121X and type 2 diabetes status by study. (PDF $145 \mathrm{~kb}$ )

\section{Competing interests}

The authors declare that they have no competing interests.

\section{Authors' contributions}

KRC and GMP wrote the manuscript. GMP and SK conceived the design of the study. KRC, AYC, JW, PN, JF, MAR, and GMP performed statistical analysis. PN, JF, MAR, DMA, SK, and GMP interpreted the results and critically revised the manuscript. SS, RM, UB, VF, RAS, DJR, MB, MIC, DMA, and SK were responsible for oversight of the contributing cohorts, and contributed to the final version of the manuscript. GMP is the guarantor of this work and takes responsibility for the integrity of the data and the accuracy of the data analysis. All authors read and approved the final manuscript.

\section{Acknowledgements}

GMP is supported by the National Heart, Lung, and Blood Institute of the National Institutes of Health under Award Number K01HL125751. SK is supported by a Research Scholar award from the Massachusetts General Hospital $(\mathrm{MGH})$, the Howard Goodman Fellowship from $\mathrm{MGH}$, the Donovan Family Foundation, R01HL107816, and a grant from Fondation Leducq. The content is solely the responsibility of the authors and does not necessarily represent the official views of the National Institutes of Health. The authors do not report any conflict of interest.

\footnotetext{
Author details

'Department of Medicine, Massachusetts General Hospital, Harvard Medical School, Boston, MA 02114, USA. ²Division of Preventive Medicine, Brigham and Women's Hospital, Boston, MA 02215, USA. ${ }^{3}$ National Heart, Lung, and Blood Institute (NHLBI) Framingham Heart Study, Framingham, MA 01702, USA. ${ }^{4}$ Department of Epidemiology, Fairbanks School of Public Health, Indianapolis, IN 46202, USA. ${ }^{5}$ Department of Medicine, Indiana University School of Medicine, Indianapolis, IN 46202, USA. ${ }^{6}$ Center for Human Genetic Research, Massachusetts General Hospital, Boston, MA 02114, USA. ${ }^{7}$ Cardiovascular Research Center, Massachusetts General Hospital, Boston, MA 02114, USA. ${ }^{8}$ Program in Medical and Population Genetics, Broad Institute, Cambridge, MA 02142, USA. ${ }^{9}$ Department of Molecular Biology, Massachusetts General Hospital, Boston, MA 02114, USA. ${ }^{10}$ Cardiovascular
}

Institute, Mount Sinai Medical Center, Icahn School of Medicine, Mount Sinai, New York, NY, USA. ${ }^{11}$ MRC Epidemiology Unit, University of Cambridge School of Clinical Medicine, Institute of Metabolic Science, Cambridge Biomedical Campus, Cambridge CB2 OSL, UK. ${ }^{12}$ Perelman School of Medicine, University of Pennsylvania, Philadelphia, PA 19104, USA. ${ }^{13}$ Center for Statistical Genetics, Department of Biostatistics, University of Michigan, Ann Arbor, MI, USA. ${ }^{14}$ Wellcome Trust Centre for Human Genetics, University of Oxford, Oxford, UK. ${ }^{15}$ Oxford Centre for Diabetes, Endocrinology, and Metabolism, Churchill Hospital, University of Oxford, Oxford, UK. ${ }^{16}$ Oxford NIHR Biomedical Research Centre, Churchill Hospital, Oxford, UK.

${ }^{17}$ Department of Genetics, Harvard Medical School, Boston, MA 02115, USA.

${ }^{18} 801$ Massachusetts Ave, Crosstown Center, Third Floor, Boston, MA 02118 , USA.

Received: 10 October 2015 Accepted: 22 January 2016

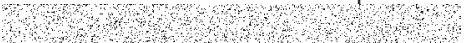

References

1. Yi P, Park JS, Melton DA. Betatrophin: a hormone that controls pancreatic beta cell proliferation. Cell. 2013;153(4):747-58

2. Gusarova V, Alexa CA, Na E, Stevis PE, Xin Y, Bonner-Weir S, et al. ANGPTL8/Betatrophin does not control pancreatic beta cell expansion. Cell. 2014;159(3):691-6.

3. Yi P, Park JS, Melton DA. Perspectives on the Activities of ANGPTL 8/ Betatrophin. Cell. 2014;159(3):467-8

4. Wang Y, Quagliarini F, Gusarova V, Gromada J, Valenzuela DM, Cohen JC, et al. Mice lacking ANGPTL8 (Betatrophin) manifest disrupted triglyceride metabolism without impaired glucose homeostasis. Proc Natl Acad Sci U S A. 2013;110(40):16109-14

5. Quagliarini F, Wang Y, Kozlitina J, Grishin NV, Hyde R, Boerwinkle E, et al. Atypical angiopoietin-like protein that regulates ANGPTL3. Proc Natl Acad Sci U S A. 2012;109(48):19751-6.

6. Peloso GM, Auer PL, Bis JC, Voorman A, Morrison AC, Stitziel NO, et al. Association of low-frequency and rare coding-sequence variants with blood lipids and coronary heart disease in 56,000 whites and blacks. Am J Hum Genet. 2014;94(2):223-32.

7. Pirinen M, Lappalainen T, Zaitlen NA, Dermitzakis ET, Donnelly P, McCarthy $\mathrm{Ml}$, et al. Assessing allele-specific expression across multiple tissues from RNA-seq read data. Bioinformatics. 2015;31(15):2497-504

8. Rivas MA, Pirinen M, Conrad DF, Lek M, Tsang EK, Karczewski KJ, et al. Human genomics. Effect of predicted protein-truncating genetic variants on the human transcriptome. Science. 2015;348(6235):666-9.

9. Muntendam P, McCall C, Sanz J, Falk E, Fuster V. The Biolmage Study: novel approaches to risk assessment in the primary prevention of atherosclerotic cardiovascular disease-study design and objectives. Am Heart J. 2010;160(1):49-57 e41.

10. Wessel J, Chu AY, Willems SM, Wang S, Yaghootkar H, Brody JA, et al. Low-frequency and rare exome chip variants associate with fasting glucose and type 2 diabetes susceptibility. Nat Commun. 2015;6:5897.

11. Flannick J, Thorleifsson G, Beer NL, Jacobs SB, Grarup N, Burtt NP, et al. Loss-of-function mutations in SLC30A8 protect against type 2 diabetes. Nat Genet. 2014:46(4):357-63.

12. Grove ML, Yu B, Cochran BJ, Haritunians T, Bis JC, Taylor KD, et al. Best practices and joint calling of the HumanExome BeadChip: The CHARGE Consortium. PLoS One. 2013:8(7):e68095.

13. Goldstein Jl, Crenshaw A, Carey J, Grant GB, Maguire J, Fromer M, et al. zCall: a rare variant caller for array-based genotyping: genetics and population analysis. Bioinformatics. 2012;28(19):2543-5.

14. Voight BF, Scott $\cup$, Steinthorsdottir V, Morris AP, Dina C, Welch RP, et al. Twelve type 2 diabetes susceptibility loci identified through large-scale association analysis. Nat Genet. 2010:42(7):579-89.

15. Purcell S, Cherny SS, Sham PC. Genetic Power Calculator: design of linkage and association genetic mapping studies of complex traits. Bioinformatics. 2003:19(1):149-50. 